\title{
Metabolic newborn screening: between past and future
}

\author{
M.A. Donati *, L. Padrini, G. La Marca, E. Pasquini \\ Metabolic and Muscular Diseases Unit, Department of Neurosciences, Anna Meyer Children's Hospital, Florence, Italy
}

\section{A R T I C L E I N F O}

\section{Keywords:}

Newborn screening

Tandem mass spectrometry (MS/MS)

\begin{abstract}
A B S T R A C T
The introduction of new technologies such as tandem mass spectrometry (MS/MS) has lead to a major revolution in newborn screening for metabolic diseases: from "a test for a disease" (i.e. Guthrie test) to "a test for many diseases". On a single drop of blood it is now possible to analyze and quantify various metabolites, not only acylcarnitines and amino acids, but also adenosine and 2'-deoxyadenosine (for the detection of ADA-SCID) and lysosomal enzymes. Therefore a great potential can be seen in near future for the diagnosis of diseases for which there may be a specific and effective therapy.
\end{abstract}

(c) 2013 Elsevier Ireland Ltd. All rights reserved.
The introduction of new technologies such as tandem mass spectrometry (MS/MS) has lead to a major revolution in newborn screening programs: from the old wiew "a test for a disease" (i.e. Guthrie test) to the new concept "a test for many diseases". Using a single drop of blood on Guthrie card (DBS, dried blood spot), indeed, it is now possible to search and quantify various metabolites (acylcarnitines, amino acids) at the same time, thus diagnosing numerous metabolic diseases: organic acidurias, fatty acids beta-oxidation defects, aminoacidopathies, urea cycle defects. From the late Nineties newborn screening programs experiences in various Countries (Australia, USA, United Kingdom, Germany) made possible the diagnosis of metabolic diseases in neonatal age, often in a pre-symptomatic phase [1].

These diseases are rare individually, but show a high frequency when considering them as a group. If not correctely diagnosed, they can lead quickly to death, or cause severe neurologic impairment. Early diagnosis by newborn screening allows the begin of the therapy in a pre-symptomatic phase, than to performing a genetic counseling. For these group of diseases, therapy consists in avoiding prolonged fast (i.e. beta oxidation defects) and/or following a dietary regime (i.e. low provision of natural protein or fat) and, in some cases, supplementation with specific formulas. Other possibles therapies are vitamines and/or drugs [2].

After a pilot study performed in 2002, Metabolic diseases Unit at Meyer Children's hospital in Florence started to manage the neonatal screening program using MS/MS since November 2004, for all the babies born in Tuscany. Since 2010, we also provide neonatal newborn screening for all the babies born in Umbria, with a total amount of 45,000 newborns screened per year. To date, over 350,000 newborns had been analyzed, with a diagnostic rate of $1: 1800$. In our experience, some diseases considerated rare or non esistent show conversely an high incidence in italian population. This is the case, for istance, of MCAD

\footnotetext{
* Corresponding author.
}

(medium chain acylCoA dehydrogenase) deficiency. This condition can be associated to death after an acute illness, i.e. for Reye syndrome, or even to Sudden infant death syndrome (SIDS). In non-screened newborns and childrens, it is possible to hypotize a missed diagnosis whenever a sudden and unexpected death occurs. In Tuscany and Umbria (two regions in central side of Italy) MCAD deficiency shows an incidence of $1: 27,000$ screened newborns; diagnosed infants maintain to date good clinical condition by following a simple dietary regime (regular meals and avoidance of prolonged fasting, expecially during acute infectious illness). Newborn metabolic screening programs are at the moment carried on in numerous others italian regions, with full or partial coverage: Liguria, Emilia Romagna, Campania, Sardinia, Lazio and Sicily.

Moreover, newborn screening in LC-MS/MS allows the diagnosis of some inherited or acquired metabolic defects in the mother, i.e. sistemic carnitine's defect (which can be associated to cardiomyopathy) so maternal diagnosis can be considered another beneficial effect of newborn screening programs. This is the case of maternal methilmalonic acidaemia with hyperomocisteinaemia due to vitamine B12 deficit. Propionylcarnitine (C3) is a metabolite included in newborn screening programs for the detection of propionic/ methilmalonic acidaemia and disorders of cobalamine metabolism. In mothers, vitamin B12 deficiency may be due to malabsorption or vegan diet. In newborns and infants, cobalamin deficiency is mainly due to maternal vitamin deficiency and may be worsened by prolonged exclusive breastfeeding. Severe haematologic, metabolic and neurological disorders may occur in infants exclusively breastfeed by mothers who follow a strict vegetarian diet. We underline the importance of newborn screening with second tier test (search for free methilmalonic acid on DBS) and combined analysis of acylcarnitines and omocisteine on mother's blood in all cases of elevated C3. This approach allows a properly diagnosis of unrecognized vitamin B12 deficiency in the mother. Therapy with i.m. hydroxocobalamin in newborn and in the mother may prevent potential damages. There is a debate on how many and which diseases should be screened, but certainly the availability of 
an effective therapy in early stage is an important prerequisite for introducing a disease in the panel. In 2006, The American College of Medical Genetics (ACMG), a group made up of specialists in public health, health policy, ethics, and other sectors involved in neonatal screening, carried out a rewiew of the literature relative to neonatal screening and indicated, among diseases that could be considered for the inclusion in screening programs, some lysosomal storage diseases (LSD) such as mucopolysaccaridosis type I (MPS I), Fabry disease, Pompe disease and Krabbe disease [3]. Arguments in favor of the inclusion of LSD in newborn screening panels have been the increasing availability of therapies (i.e. enzyme replacement theraphy, bone marrow transplant) [4] and the probable underestimation, in some cases very substantial, of the LDS' real prevalence in world population [5]. In recent years, the widespread use of DBS combined with MS/MS has constituted a turning point for the analysis of lysosomal enzymes associated to some storage diseases (Fabry, Gaucher, Pompe, Krabbe, Niemann-Pick A/B diseases and Mucopolysaccharidosis I). It's a strong, highly sensitive, rapid, cheap and non ivasive method; it's suitable for screening projects both in the neonatal population that in high-risk populations, both for the targeted analysis of patients with specific symptoms. A single DBS allows multiple enzymatic analysis and, moreover, a high number of samples can be analyzed simultaneously. In our laboratory whe have therefore developed methods of enzyme assay using MS/MS on DBS for the diagnosis of some LSD suitable to treatment: Fabry disease ( $\alpha$-galactosidase), Pompe disease ( $\alpha$-glucosidase) and Mucopolysaccaridosis type I ( $\alpha$-iduronidase) [6]. Numerous experiences of newborn screening for lysosomal diseases have already been reported in international literature. A recent study of MS/MS enzyme assay for Fabry, Pompe and MPS I disease on 100,000 DBS from anonymous newborn showed a combined prevalence of 1:7500 (low enzyme activity confirmed with genetic molecular test). [7]. The study concludes that simultaneous enzyme assay on MS/MS can be introduced into neonatal screening panel, and that this method has a predictive value equal to or better comparing with the methods currently used for non-lysosomal diseases. In 2006, an italian study estimated the incidence of Fabry disease diagnosed by newborn screening (fluorimetric test on DBS) to be $1: 3100$ (on a population consisting of 37,104 male infants) [5]. In Taiwan, Pompe disease was diagnosed by screening with a prevalence of $1: 33,333$ (on a population of 132,000 screened newborns; age at diagnosis 9-22 days) [8]. From follow-up analysis of patients with Pompe disease there is an evidence that $100 \%$ of the patients diagnosed by screening in a presymptomatic phase are ventilator-free; on the contrary, $50 \%$ of the patients diagnosed in a symptomatic phase (even those diagnosed within six months of age) are ventilator-dependent [9]. Regarding European experiences, a neonatal screening on DBS for four LSD (Pompe, Fabry, Gaucher and Niemann Pick A/B) was conducted in Austria from January 2010 in 34,736 newborns [10]. The study identified positive samples which were then correlated with a specific condition: 2 cases of Gaucher disease, 4 cases of Pompe disease and 9 cases of Fabry disease, with a complessive prevalence of $1: 2315$ births. Neonatal screening by LC-MS/MS requires great organizational commitment, and high laboratoristic and clinical experience. A positive test must be followed by a rapid newborn recall, for laboratory confirmation test and clinical treatment and follow up. New test and protocols (i.e. for low birthweight newborns) have been developed and can reduce the possibility of false positive and false negative tests, thus avoiding unnecessary stress or misdiagnosis.

\section{Conflict of interest}

The authors have no conflict of interest to declare.

\section{References}

[1] Wilcken B, Wiley V, Hammond J, Carpenter K. Screening newborns for inborn errors of metabolism by tandem mass spectrometry. $\mathrm{N}$ Engl J Med 2003;348:2304-12.

[2] Saudubray JM, Van Den Berghe G, Walter JH. Inborn Metabolic Diseases: Diagnosis and Treatment, Fifth edition. Berlin: Springer-Verlag, 2012.

[3] American College of Medical Genetics Newborn Screening Expert Group. Newborn screening: toward a uniform screening panel and system - executive summary. Pediatrics 2006;117:S296-307.

[4] Desnick RJ. Enzyme replacement and enhancement therapies for lysosomal diseases. J Inherit Metab Dis 2004;27:385-410.

[5] Spada M, Pagliardini S, Yasuda M, Tukel T, Thiagarajan G, Sakuraba H, Ponzone A, Desnick RJ. High incidence of later-onset Fabry disease revealed by newborn screening. Am J Hum Genet 2006;79:31-40.

[6] La Marca G, Casetta B, Malvagia S, Guerrini R, Zammarchi E. New strategy for the screening of lysosomal storage disorders: the use of the online trapping-and-cleanup liquid chromatography/mass spectrometry. Anal Chem 2009;81:6113-21.

[7] Scott CR, Elliott S, Buroker N, Thomas LI, Keutzer J, Glass M, Gelb MH, Turecek F. Identification of infants at risk for developing fabry, pompe, or mucopolysaccharidosis-i from newborn blood spots by tandem mass spectrometry. J Pediatr 2013;163(2):498-503. doi: 10.1016/j.jpeds.2013.01.031 [Epub 2013 Mar 1].

[8] Chien YH, Lee NC, Thurberg BL, Chiang SC, et al. Pompe disease in infants: improving the prognosis by newborn screening and early treatment. Pediatrics 2009;124(6):1116-25.

[9] Chien YH, Hwu WL, Lee NC. Pompe disease: early diagnosis and early treatment make a difference. Pediatr Neonatol 201354(4):219-27. doi: 10.1016/ j.pedneo.2013.03.009 [Epub 2013 Apr 28].

[10] Mechtler TP, Satry S, Metz TF, De Jesus VR, Greber-Platzer S, Pollak A, Herkner KR, Strenbel B, Kasper DC. Neonatal screening for lysosomal storage disorders: feasibility and incidence from a nationwide study in Austria. Lancet 2012;379(9813):335-41. 